\title{
Observaciones sobre la función de las tablas de Baja California
}

\author{
Gianfranco Cassiano V. \\ Escuela Nacional de Antropología e Historia
}

\section{Resumen}

El presente escrito reúne y analiza determinada información acerca de las tablas que fueron halladas en esta región, proporcionando datos de estos objetos tanto de carácter morfológico, etnográfico, como de su posible función social. Es fundamental el estudio de los procesos productivos donde interviene el artefacto, como objeto y como medio de trabajo, así como analizar la morfología, el tipo del sitio y el contexto arqueológico, su asociación con el contexto, la filiación cultural y su lugar en el tiempo. En suma, estas tablas son la expresión reciente de una necesidad más antigua: la de reproducir y reforzar las relaciones sociales de producción y las bases del prestigio dentro del grupo, utilizando los patrones de descendencia y la estructura de parentesco.

Palabras clave: prehistoria, tablas, patrones de descendencia, estructura de parentesco, sitios arqueológicos, indígenas de Baja California, "tótems".

\begin{abstract}
This project gathers and analyzes specific information about certain wood planks found in this region, while offering morphologic, ethnographic information, about these objects, as well as its potential social function. It is critical to perform a research regarding the use and purpose of this artifact within the productive processes as an object; but also to study on it as a working tool, to analyze its morphology, the site where they were found, its archeological context, its association to this context, its cultural relationship, and its place in an specific frame of time. In summary, these wood planks are the recent expression of an even older need: to reproduce and reinforce social relations of production and the foundations for prestige in the middle of a social group by using the descendant patterns and kinship structures.
\end{abstract}

Keywords: pre-history, wood planks, descendant patterns, kinship structure, archeological sites, Indians of Baja California, "totems". 


\title{
OBSERVACIONES SOBRE LA FUNCION DE LAS TABLAS DE BAJA CALIFORNIA
}

\author{
Por \\ Gianfranco Cassiano V.*
}

Hace algunos años, los estudiosos de Baja California dedicaron su atención a unos objetos característicos de la prehistoria de la península: las tablas. Se trata de una decena de objetos de madera rectangulares, cuyo largo promedio es alrededor de $80 \mathrm{~cm}$ y cuyo ancho y espesor son variables.

A comienzos de los setenta se publicaron varios artículos de diferentes autores, enfocados más hacia las tablas mismas que hacia el contexto en el cual se encontraban, quedando algunos en un nivel exclusivamente descriptivo morfotipológico (Davis, 1968: 52-55; Massey, 1972: 25-34; Williams, 1975). Asimismo, la utilización de las fuentes históricas, casi siempre de segunda mano y en traducciones al inglés, se limitó al aspecto del uso inmediato, y la función social de las tablas se vio generalmente dentro de una supuesta superestructura religiosa (Hedges, 1973: 5-21). El único autor que trató de ir más allá de este nivel fue Ritter, quien sin embargo, utiliza de manera muy vaga conceptos como difusión y adaptación (Ritter, 1974: 29-37).

Para el arqueólogo, cuyo objeto de estudio es la cultura material, la función se relaciona con características formales como la morfología del implemento, el tipo de sitio y de contexto arqueológico, su asociación con el contexto, la filiación cultural y la ubicación cronológica. Igualmente fundamental es el estudio de los procesos productivos donde interviene el artefacto, primero como objeto de trabajo y después como medio de trabajo. El propósito de este escrito es analizar la información que se posee para cada uno de los aspectos mencionados, básicamente la de las fuentes históricas, la arqueología de campo y la de gabinete.

Localización geográfica. Ocho de las diez tablas provienen de la parte norte de la península: cuatro de la zona de San Faustino, tres del Valle de la Trinidad y una es de procedencia desconocida. Las dos restantes se hallaron en la parte central: una en los alrededores de La Purísima y otra en bahía Coyote (figuras 1 a 4).

Morfología: materia prima y forma. La materia prima utilizada era madera, prefiriéndose el pino y el álamo en el norte, y el mezquite en el centro. Esta diferencia puede deberse a la disponibilidad local de árbolescon las características requeridas para este tipo de trabajo.

En cuanto a la forma, otros autores ya subrayaron las notables diferencias entre el norte y el centro, observando la presencia de agarradera y el uso generalizado de la decoración pintada en el norte, y las perforaciones en la

\footnotetext{
- Arqueólogo catedrático de la Escuela Nacional de Antropología e Historia.
} 


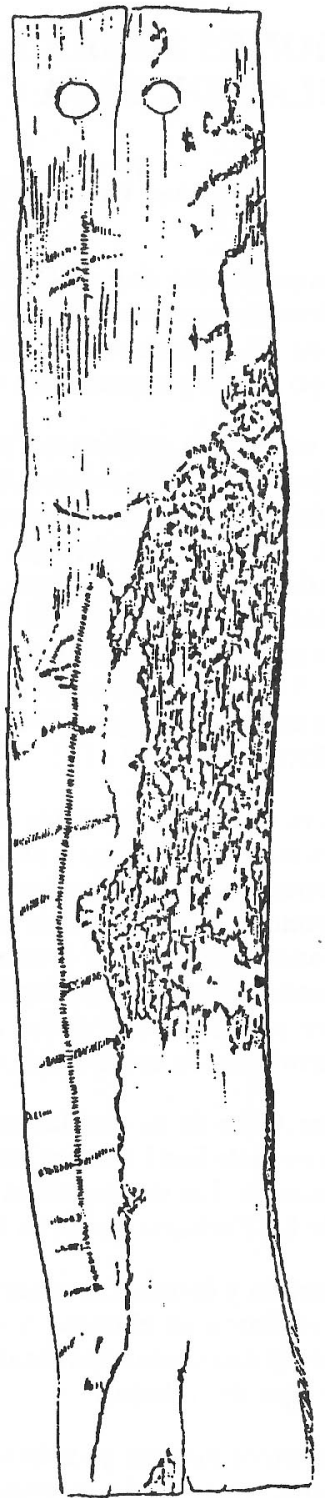

FIGURA 1. Tabla de bahía Concepción. 

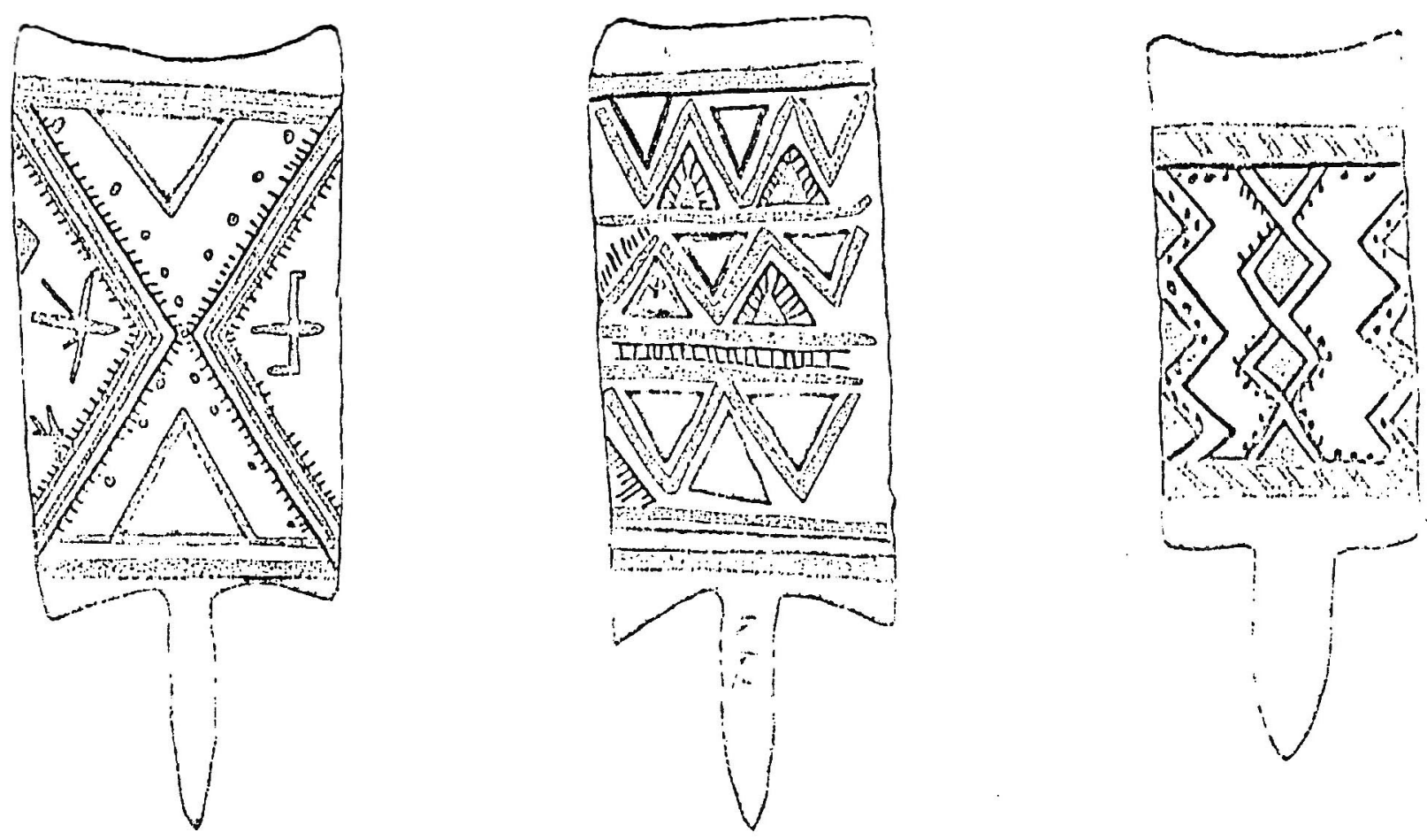

FIGURA 2. Tablas de San Faustino 

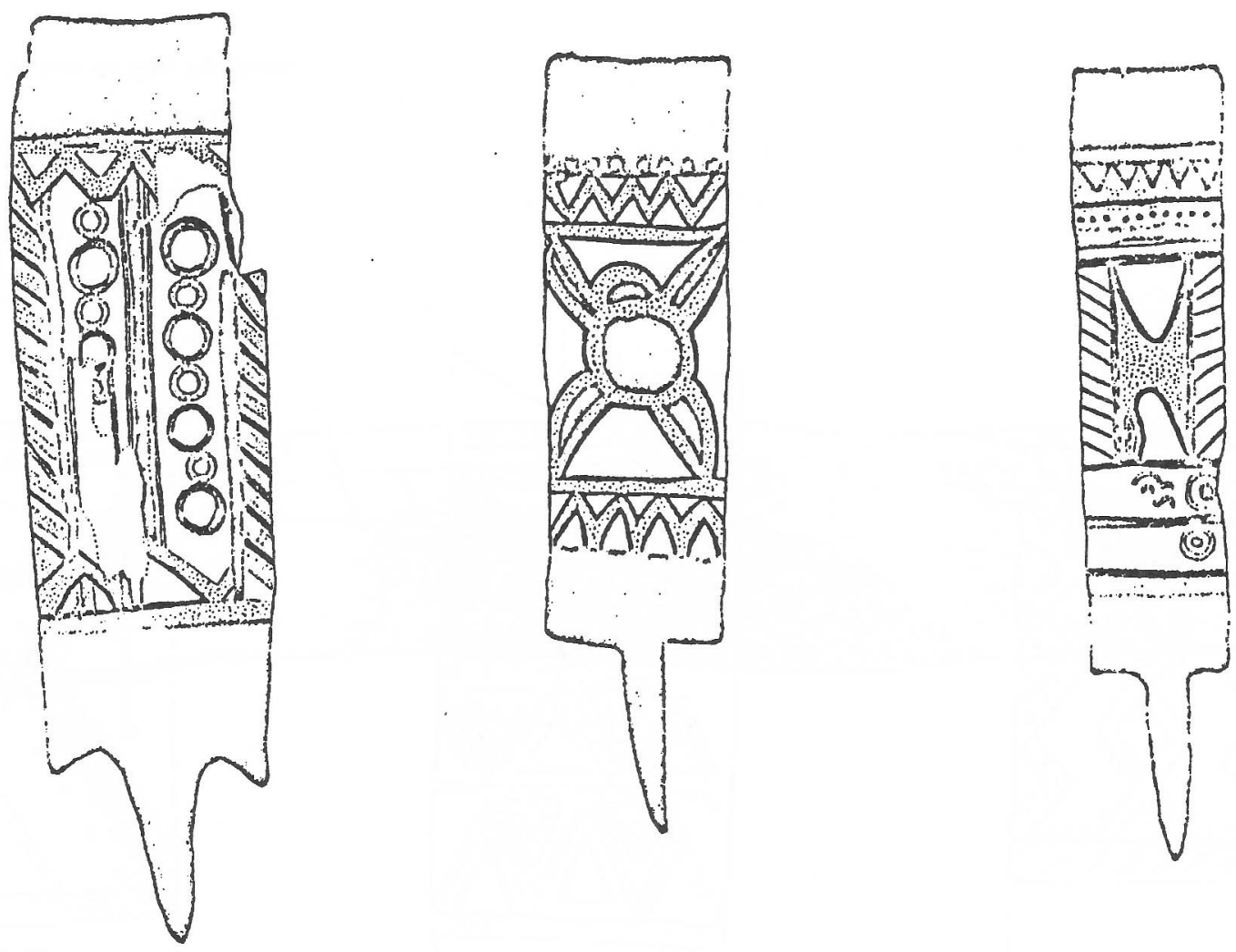

FIGURA 3. Tablas halladas en Valle de la Trinidad. 
Gianfranco Cassiano V.

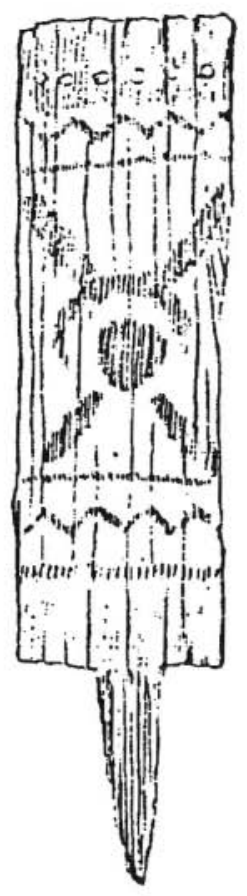

A

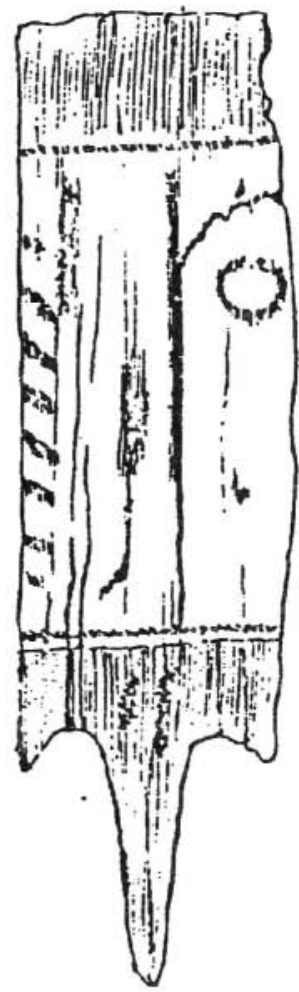

B

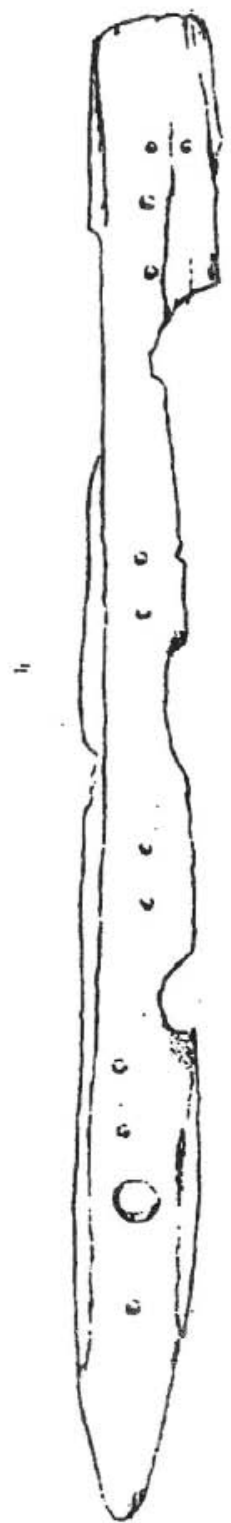

c

FIGURA 4. Tablas (C-La Purísima). 
región central. Preferimos no utilizar las dimensiones como un atributo morfológico discriminatorio, puesto que los especímenes hallados representan una muestra insuficiente y poco documentada, sobre todo para el centro de la península, y las fuentes no dan información utilizable. Por ejemplo, Consag atribuye las diferencias en las dimensiones al tipo de madera empleado: "Los vecinos al océano tienen las tablas más anchas; porque se valen de unos pinos que hallan en la playa"(Ortega, 1944).

Tipo de sitio y de contexto arqueológico. Todos los hallazgos de tablas han sido casuales y se han dado en cuevas o abrigos, lo cual es lógico porque en estos lugares los materiales perecederos tienen mayor probabilidad de conservarse, mientras que los que se encuentran en sitios abiertos han sufrido y siguen sufriendo.una severa destrucción por causas naturales y humanas. Por otro lado, se podría suponer que las cuevas fueron los lugares de utilización de las tablas, o que éstas últimas eran ofrendas que allí depositaban. Es también posible que hayan servido de escondites, para evitar que los misioneros las destruyeran (Aschmann, 1968: 46-51).

El tipo de contexto arqueológico se desconoce, puesto que los hallazgos nunca se han dado en el curso de una excavación controlada.

Asociación y filiación cultural. La única asociación indudable se encontró en la superficie de una cueva en San Faustino, Baja California; estaba constituida por cuatro tablas pintadas, con agarradera y cinco pipas - lo que señalaría la filiación yumana del conjunto - y han sido interpretadas como ofrenda (Hedges, 1973). Las tablas halladas en el Valle de la Trinidad están en una colección privada junto con otros objetos del mismo valle que pueden ser atribuidos a los kiliwas históricos, pero la asociación de las tablas con éstos es muy dudosa.

Cronología. No se ha hecho ningún fechamiento absoluto, así que sólo podemos situar las tablas en los tiempos históricos, posteriormente a la llegada de los misioneros; sin embargo, seguramente se usaban desde tiempos anteriores. Según Hedges, de las cuatro tablas de San Faustino, por lo menos una tiene huellas de una sierra y tres de ellas serían bastante recientes (Hedges, 1973).

Procesos productivos: tabla como objeto de trabajo. Tanto los misioneros como los investigadores coinciden sobre el empleo de instrumentos primitivos para la fabricación de casi todas las tablas, pero hay que recordar que desde los primeros contactos con los españoles, los indígenas aprendieron a utilizar los cuchillos de acero que recibían en regalo y que, de ser utilizados, facilitarían los primeros pasos del proceso de trabajo, recurriendo a la tecnología tradicional para dar el acabadofinal. Una situación parecida se dio con los seris hace algunos años, en sus primeros trabajos de talla de palofierro (Ryerson, 1976: 119-136).

Que nosotros sepamos, en los sitios arqueológicos explorados hasta la fecha no se ha encontrado madera trabajada en forma tabular, pero es evidente, por otros restos, que losindígenas dominaban las técnicas de talla de la madera y las podían adaptar a la fabricación de otros implementos. Existe la posibilidad de 
que la idea de la tabla haya sido adoptada por los indígenas, antes o después del contacto con los españoles, a partir de las tablas que encontraban tiradas en la playa a raíz de naufragios, así que en muchos casos no necesitaban fabricarlas enteramente, sino que con sus instrumentos líticos y de concha, les daban el acabado final deseado.

Procesos productivos: tabla como medio o instrumento de trabajo. No se posee prácticamente información, pero se formularán hipótesis cuando se discuta la función de las tablas.

Puesto que todos los relatos sobre el temase refieren a la porción central de la península, tradicionalmente el área cochimí, a ésta hay que referir directamente nuestra interpretación y consideraciones generales que, por otro lado, creemos pueden proporcionar hipótesis válidas también para explicar la función de las tablas de Baja California.

Función de las tablas. Hablaremos de función a dos niveles diferentes y correlacionados: la función inmediata y la función mediata.

La función inmediata. Ha sido descrita por algunos misioneros de cuyos relatos creemos conveniente citar las partes más importantes.

Venegas, utilizando como fuente a Salvatierra dice que ". . . [los hechiceros] retiraban para esto a los niños a algunas cuevas o parajes apartados de los bosques y allá les enseñaban a formar ciertas figuras en unas tablas; y aprendidas aquéllas, les enseñaban otras al modo que se hace en las escuelas, para enseñar a escribir a los niños. . . al llegar el tiempo en que los indios se ocupaban en la cosecha de las pitahayas, se desaparecían del Real de Loreto todos los niños. . "(Venegas, 1944:94).

Según este autor, las tablas se usaban entonces para impartir algún tipo de enseñanza a todos los niños (probablemente sólo a los varones)durante la fiesta de la pitahaya, ocasión en la cual se reunían varias rancherías. Esto haría pensar en un rito de la pubertad para varones, pero no va muy de acuerdo con la afirmación de que participaban todos los niños, es decir, sin importar la edad. Tal ceremonia se llevaba a cabo a escondidas del misionero y en un lugar alejado del de reunión de los grupos. Por otrolado, la mención de las cuevas nos remite a los lugares de hallazgo de las tablas.

El mismo autor también describe una reunión durante la cual “. . . [los jefes] así enloquecidos empezaban el sermón de sus Dogmas, acompañado de ademanes descompuestos y de indecentes locuras. Fingíanse inspirados de aquellos espíritus que no reconocía la nación; y en nombre de ellos les anunciaban todo lo que les dictaba su furor o interés. . . lo más regular era tener en las manos unas tablillas formadas con gran trabajo, por falta de herramientas, del corazón del mezquite o de otro palo, que llaman uña de gato, en las cuales tenían pintadas disparatadas figuras que decían ser copias legítimas de la Tabla, que al irse al cielo les dejó el Espíritu Visitador; y éstas mismas eran las que enseñaban los de Loreto en su escuela secreta a los niños. .."(Venegas, 1944: 95). 
Aparentemente, se trata de una situación distinta de la anterior, pero Venegas afirma que en ambos casos se usaba el mismo tipo de tabla. Otro dato importante es la mención de los tipos de madera empleados y de la presencia de pinturas en las tablas.

Clavijero, que también utiliza a Salvatierra, parece mezclar las dos situaciones y relata que “. . estos guamas o charlatanes escogían entre los niños aquellos que les parecían más astutos e idóneos para tal oficio, yllevándolos a los lugares más recónditos dẹ los bosques, los iban adiestrando en sus misterios, y especialmente en hacer en ciertas tablitas algunas figuras misteriosas, que fingían ser copias de las que, según decían, les había dejado al retirarse el espíritu visitador. Estas tablitas eran los libros en que fingían leer la naturaleza de las enfermedades, los remedios a ellas convenientes, las futuras mutaciones del aire y aun el destino de los hombres . . ."(Clavijero, 1975: 67).

Lo interesante de esta versión es la asociación de las tablas con la práctica de la medicina tradicional y la actitud del autor hacia esta última. Por otro lado, aquí el autor parece señalar criterios selectivos para la participación de los niños.

Consag afirma que ". . . cuando se juntan muchas poblaciones para celebrar algún convite, cada una viene cargada con el cestillo de su ídolo, delante de cada uno clavan su tabla más ancha o más estrecha, o larga o corta, según fuera la madera que tuvieron..." (Ortega, 1944: 404)

Nuevamente encontramos relacionado el uso de la tabla con una festividad; por lo que sabemos, tanto en el norte como en el centro-sur de la península las reuniones de varias bandas estaban ligadas a la explotación de algún recurso estacionalmente abundante y probablemente a la realización de actividades, por ejemplo las bélicas, que trascendían el nivel mínimo numérico de la banda.

En cuanto a la función de la tabla, se ha pensado que ésta fuera como un altar, pero nosotros pensamos en una especie de tótem, como veremos más adelante. Importante también es la mención de los ídolos, que no se encuentra frecuentemente en las descripciones de las prácticas rituales de los californianos.

Sales también consigna el uso de las tablas durante reuniones: “. . . luego saca [el jefe] unas tablas pintadas con mil figurones los que representan los hombres más hábiles que han tenido ellos, los mejores curanderos, los más valientes, los más corredores y los más fuertes, y sólo de éstos forma unos elogios muy sobresalientes pero siempre añade, que él es más que todos. . ."(Sales, 1960: 48).

Sales describe también otro tipo de tabla, usada en la misma reunión: “. . . a más de estas tablas hay otra que tendrá una vara de largo, y media de ancho; en medio tiene agujero, y de cuando en cuando [el jefe] mete y saca la lengua, y todos se ríen descompasadamente. . ." (Sales, 1960: 48). 
Aschmann lo interpretó como la simbolización del acto de hablar por parte del objeto (Aschman, 1968) y Ritter retomó esta idea, atribuyendola función de máscara a la tabla encontrada por élen bahía Concepción, por el hecho de tener dos hoyos paralelos en una extremidad (Ritter, 1974).

Taraval reporta que en las perforaciones se colocaban adornos de plumas (Williams, 1975). Esta podría ser la interpretación más correcta también en el caso de la tabla hallada por Ritter, que además tiene una extremidad: la opuesta a la perforada, más delgada, quizá con el fin de facilitar su hundimiento en el suelo. Esta misma finalidad pudieron tener las agarraderas de las tablas encontradas en el norte de la península.

Resumiendo, las fuentes evidencian tres funciones inmediatas, aparentemente distintas de las tablas, sin dejar completamente en claro si existian diferencias morfológicas importantes implicadas.

1. Tablas para impartir enseñanza a los niños, que estaban pintadas.

2. Tablas usadas en las ceremonias públicas, que podían tener pintura $u$ hoyos, o quizá las dos cosas al mismo tiempo.

3. Tablas como altares o "totems", que también podían tener tanto pintura como hoyos.

Lo que las diferentes versiones tienen en común es que las tablas eran utilizadas por los hombres durante las reuniones de varias "rancherías" y eran manejadas por el "jefe" o por el "hechicero" del grupo. Es posibleque en muchos casos, este doble papel fuera cumplido por la misma persona.

Basándonos conjuntamente en las fuentes antes citadas, es muy plausible pensar que uno o varios tipos de tablas se fabricaban y se decoraban para representar los elementos de identificación de un grupo frente a los otros, y que en ellas se quería expresar, a través de símbolos pictóricos, la descendencia patrilineal, misma que se utilizaba como criterio de diferenciación. Este patrón de descendencia es común en las sociedades de cazadores-recolectores organiradas en bandos (Service, 1973).

La función mediata. Por su función inmediata, las tablas no intervienen directamente en ningún proceso productivo de alimentos, más bien indirectamente, como elementos reproductivos de la estructura social. En este sentido, la cnseñanza impartida a los niños pudo tener como objetivo el delimitar las actividades productivas importantes del grupo, el papel masculino en ellas y el valor de la aportación individual dentro de las habilidades específicas de cada quicn, estableciendo criterios de valoración de tales habilidades y, por ende, de justificación de situaciones de prestigio.

I a idea de la tabla como copia de la dejada por el espiritu visitador, si por un lado nos sugiere la posibilidad del sincretismo, por el otro se puede explicar por la estrecha relación que debió existir entre la cosmogonía, la cosmología y la explicación de los fenómenos de la naturaleza. Del conocimiento de estos 
fenómenos y de la validez de la explicación depende en última instancia la capacidad de sobrevivencia de los grupos. De tal forma, las tablas serían producto y expresión de la capacidad adaptativa de la sociedad, siendo factor importante en el funcionamiento de la organización en bandas. También es posible que, dentro de un mismo grupo residencial, existieran varias tablas en relación con cada familia restringida.

Lo anterior nos introduce a otra discusión, sobre el "valor"de las tablas. Este se ha querido medir por la complejidad implicada en el proceso de producción y por la cantidad de tiempo de trabajo necesaria, en relación con otros procesos de manufactura (Aschmann, 1968). Según la afirmación de Consag "estas tablas son a su barbaridad [de los indios] de mucho aprecio, tal vez porque les cuesta mucho tiempo y más trabajo que se puede inferir fácilmente, con saber que sin más hierro que una piedra o pedernales afilados, han de desbastar el palo, labrarlo y pulirle, hasta llegar a lo delgado de una tabla"(Ortega, 1944: 404).

Suponiendo que en todos los casos la fabricación de las tablas era llevada a cabo por los indígenas, éstas se han visto como un producto sofisticado de sociedades que se caracterizan por la sencillez y la rusticidad de sus implementos materiales. Hay que hacer notar a este propósito, que nuestra concepción de valor del tiempo y del trabajo es bastante diferente de la de un cazadorrecolector; para éste, el valor del objeto debió residir másen su utilidad (valor de uso) que en el esfuerzo implicado en su obtención; pero sí es posible que tal esfuerzo, es decir la energía utilizada, fuese a veces medida de utilidad.

Según Consag, en algunos casos las tablas eran parte del intercambio de regalos que los indígenas tenían con los españoles cuando se encontraban: “. . . en las lomas se vio bastante gente que de una $€ n$ otro corrían, y a carrera llegaron de cuatro y de seis en varios trozos con mescales, tablas, plumas y sus armas, que se les cambiaron ..."(Ortega, 1944: 406).

De lo anterior se deduce que podían desprenderse de ellas, aunque en circunstancias excepcionales y movidos por una motivación muy poderosa. En este sentido cuenta Venegas: "Ofreciósele al padre cómo aprendiesen la doctrina y le trajesen las tablillas, chacuacos y cabelleras. Respondieron todos que la doctrina ya la sabían, y que allí traían las tablillas y demás instrumentos, porque sabían que sin eso no los había de bautizar". (Venegas, 1944: 249). Por otro lado, no sabemos si tales tablas tenían la misma función de las que citamos anteriormente, ya que como se ha dicho, dentro de una misma forma general se pudieron dar funciones diferentes.

Bajo la influencia de la visión de los misioneros y por datos etnográficos recientes (Aschmann, 1968) se ha llegado a pensar en las tablas simplemente como elementos del mundo mágico-religioso de los cazadores-recolectores y como parte del instrumental del "hechicero". Esta asociación se puede deber a que los shamanes, como depositarios de la tradición y de la ideología del grupo, ofrecieron mayor resistencia a ser aculturados, no sólo porque esto implicaba una pérdida concreta de prestigio de su parte, sino porque la 
cultura invasora les planteaba un camino desconocido y una desviación del nivel adaptativo alcanzado.

Por otro lado, los misioneros no querían eliminar las tablas sólo para reemplazarlas con las imágenes del cristianismo; más bien, si nuestra interpretación es válida, en ellas pretendían destruir uno de los elementos importantes de la identidad étnica, para facilitar la reagrupación de los indígenas y el cambio de su modo de producción, de cazadores-recolectores a agricuitores. Con esto no queremos afirmar que los misioneros asociaron directamente las tablas con los aspectos mencionados de la estructura social pero, en su simple nivel de causaefecto, seguramente se daban cuenta de que la entrega de la tabla era símbolo de sumisión y de renuncia, por parte de los indígenas, de su tradicional modo de vida.

Area de distribución. Se han encontrado tablas en el norte y en el centro de la península, con las diferencias morfológicas ya mencionadas. Para explicar las similitudes, Ritter recurre a un criterio difusionista en sentido norte-sur, planteando que la aceptación de tal elemento se debería a la similitud en la adaptación general de los diferentes grupos. Según el mismo autor, las diferencias estarian ligadas a diferencias étnicas y, en segundo lugar, a diferentes funciones dentro del mismo grupo (Ritter, 1974).

En cuanto al primer punto, el de la difusión, aun estando de acuerdo con las implicaciones adaptativas de las tablas, resulta difícil pensar en la difusión de un rasgo aislado, ya que por las fuentes históricas (Clavijero, 1975) sabemos que a lo largo de la península existieron, en la época misional, etniasque presentaban afinidades en algunos aspectos de la cultura material y de la práctica económica, así como diferencias lingüísticas y de estructura social. Por éstas últimas, la introducción de nuevos elementos en el ámbito de las relaciones de producción debe presuponer el empuje de un estímulo cultural muy fuerte y la adaptación dentro de una nueva función ya existente más que la creación de una nueva función. Tal estímulo pudo haber sido la llegada de los españoles; las poblaciones indígenas, que se caracterizaban por una marcada y agresiva territorialidad en relación con la captación de recursos estacionales y dispersos, empezaron a sufrir un proceso de reagrupación por la organización de los españoles en torno a nuevas actividades productivas, y la mayor cohesión que de allí resultó pudo alterar la cantidad y la calidad del flujo de información. Entonces es posibleque, antes de que se completara el proceso de aculturación, el área de distribución de las tablas hubiera aumentado con respecto a los tiempos anteriores a la conquista.

En cuanto a las diferencias morfológicas, si bien en parte se deben a diferentes etnias, siendo las tablas precisamente expresión de éstas, también pueden estar relacionadas con cambios en el tiempo de la función y de su contenido ideológico. Según Hedges (1973: 37-39) y Meigs (1974), las tablas encontradas en la parte norte de Baja California tenían un uso funerario; sin embargo, en las fuentes históricas no se consigna estafunción de manera directa y además, tales tablas parecen ser las más recientes cronológicamente. 
Anteriormente planteamos que dentro de un mismo grupo existieron varios tipos de tablas con diferentes funciones, pero el reconocer tales diferencias depende del conocimiento que tengamos de la cosmología y del simbolismo indígenas; es decir, no podemos simplemente basarnos en diferencias de la morfología externa, puesto que todo objeto tiene doble funcionalidad inmediata: una hacia el trabajo que tiene que cumplir y otra hacia la persona que lo está empleando; su forma es el resultado de tal duplicidad. Siendo las tablas elementos móviles y producto de grupos que, para su sobrevivencia, necesitaban ejercer cierta movilidad, algunas de sus características externas deben estar pensadas en función de su transportación y manejo y otras en función de la información social que tenían que llevar; desafortunadamente, todavía no estamos capacitados para reconocer tales características y las formas peculiares en que cada grupo. las resolvía prácticamente. Un estudio de este problema necesitaría de una extensa investigación de campo de los grupos que nos interesan, con la esperanza de encontrar un mayor númerode tablas y alcanzar el tamaño de una muestra representativa.

En conclusión, nuestra hipótesis es que las tablas son la expresión reciente de una necesidad más antigua: la de reproducir y reforzar las relaciones sociales de producción y las bases del prestigio dentro dẹl grupo, utilizando los patrones de descendencia y la estructura de parentesco.

Como consecuencia de la represión económica e ideológica ejercida por los misioneros, que desemboca en el cambio de modo de producción, el uso de las tablas pierde su contenido adaptativo y parece limitarse al nivel de la superestructura y del simbolismo puro, como herencia tradicional en una sociedad ya diferente y vaciado de sus implicaciones en la esfera económica. Las tablas se convierten finalmente en uno de los emblemas de los indígenas, en su resistencia a ser absorbidos completamente por la otra cultura, que los había de llevar a la destrucción. 


\section{BIBLIOGRAFIA}

ASCHMANN, O. 1968. "Historical Accounts and Archaeological Discoveries: Working together two Scholarly Disciplines Enlarge our Understanding of the Extinct Indians of Baja California" Pacific Coast Archaeological Society. Quarterly, vol. 4, № 1.

CLAVIJERO, F.X. 1975. Historia de la Antigua o Baja California. Ed. Porrúa, México.

DAVIS, E. L. 1968. "Painted Wooden Tablas of Northern Baja California". Pacific Coast Archaeological Society. Quarterley, vol. 4, № 1.

HEDGES, K. 1973. "Painted Tablas from Northern Baja California". Pacific Coast Archaeological Society. Quarterly, vol. 9, № 1.

MASSEY, L.G. 1972. “Tabla and Atlatl. Two Unusual Wooden Artifacts from Baja California". Pacific Coast Archaeological Society. Quarterly, vol. 8, № 1 .

MEIGS, P., III. 1974. "Meigs on Tablas". Pacific Coast Archaeological Society. Quarterly, vol. 10, № 1.

ORTEGA, J. 1944. Maravillosa reducción y conquista de la provincia de San Joseph del Gran Nayar. Ed. Layac, México.

RITTER, E.W. 1974. “A Magico-Religious Wooden Tablet from Bahía Concepción, Baja California”. Pacific Coast Archaeological Society. Quarterly, vol. 10, № 1.

RYERSON, S.H. 1976. "Seri Ironwood Carving: An Economic View”. En: N.H. Graburn (ed.). Ethnic and Tourist Arts. Cultural Expressions form the Fourth World. University of California Press. E.U.A.

SALES, L. 1960. Noticias de la provincia de la California. Colección Chimalistac. José Porrúa Turanzas. Madrid.

SERVICE, E.R. 1973. Los cazadores. Ed. Labor, Barcelona.

VENEGAS, M. 1944. Noticia de la California y de su conquista temporal y espiritual. Ed. Layac, México.

WILliAmS, A. de Alvarez. 1975. Primeros pobladores de la Baja California. Introducción a la antropología de la península. Mexicali, B.C., México. 